\title{
ON THE GEOMETRY AND KINEMATICS OF SMOOTHLY DISTRIBUTED AND SINGULAR DEFECTS
}

\author{
MARCELO EPSTEIN AND REUVEN SEGEV
}

\begin{abstract}
A continuum mechanical framework for the description of the geometry and kinematics of defects in material structure is proposed. The setting applies to a body manifold of any dimension which is devoid of a Riemannian or a parallelism structure. In addition, both continuous distributions of defects as well as singular distributions are encompassed by the theory. In the general case, the material structure is specified by a de Rham current $T$ and the associated defects are given by its boundary $\partial T$. For a motion of defects associated with a family of diffeomorphisms of a material body, it is shown that the rate of change of the distribution of defects is given by the dual of the Lie derivative operator.
\end{abstract}

\section{INTRODUCTION}

We present below a mathematical framework for the description of the geometry and kinematics of material defects from the continuum mechanics, macroscopic, point of view. In particular, the proposed framework applies to both continuously distributed as well as singular defects and is formulated on general manifolds devoid of any metric or a parallelism structure.

Material defects, are frequently described by relative deformation of neighboring points in the material (e.g., [KA75, LK06, Sah84]). Sometimes a global point of view is adopted (e.g., [Cer99]) and defects are viewed as obstructions to the construction of a global inverse deformation. Another frequent approach (e.g., [Kon55, Nol67, Wan67, EE07]), views the existence of defects, or inhomogeneities, as an inherent consequence of the constitutive relation for a body. Following [ES12], the present framework differs from the first point of view above in the sense that the analysis involves no kinematics of the body in space. No deformations are considered and only the material structure of the body manifold is studied. The present approach differs from the theory of inhomogeneities in the sense that rather than associating the defects with a particular constitutive relation, e.g., the relation between the stress and the deformation, the material structure is given explicitly. (See somewhat similar approaches in by Toupin [Tou68] and Eringen \& Claus [EC70] who use oriented, or micromorphic media.)

2000 Mathematics Subject Classification. 74A05; 74E20; 58A25.

Key words and phrases. Continuum mechanics; ;defects; dislocations; disclinations; differential forms; singularities; de Rham currents; Frank's rules; kinematics of defects. 
For example, it is assumed that a family of Bravais hyperplanes is given by explicitly prescribing, at each point in a body, a hyperplane as well as the density of these hyperplanes. In other words, one specifies a distributed analog of the Miller indices for a family of hyperplanes. Mathematically speaking, if the body is a manifold $\mathcal{M}$ of dimension $n$, we consider in the continuous case a distribution, an $(n-1)$-subbundle of the tangent bundle, which is induced by a differential 1-form $\varphi$. Material structure of dimension $r \neq n-1$, will be prescribed by a $p$-form for $p=n-r$. Singular material structure of dimension $r$ is given in terms of a de Rham $r$-current $T$, a generalized $(n-r)$-form. Thus, for a 3-dimensional manifold, the interesting cases are $r=2$ that gives the Bravais planes at the various points and $r=1$ that gives the inclination field of directors for the theory of disclinations.

In the deformation theory of dislocations, the Burgers vector is defined using the gap that opens up between the positions of neighboring points. Here, one considers the total amount of hyperplanes that are penetrated, in one particular orientation, when a closed loop is followed. This motivates the definition of the distribution of defects as the exterior derivative $\mathrm{d} \varphi$ of the structure form $\varphi$ in the continuous case and as the boundary $\partial T$ of the structure current $T$ in the singular case.

An attempt was made here to introduce some of the relevant background on differential forms and de Rham currents. In Section 2 we briefly review the subject of distributions, subbundles of the tangent bundle, induced by a decomposable differential forms and the results pertaining to the submanifolds they may induce. In Section 3 we use these results to introduce structure $p$-forms and the corresponding exterior derivatives that represent the associated smoothly distributed defects. Section 4 presents the basic notions concerning de Rham currents and Section 5 uses these notions to introduce the singular counterpart of structure forms and continuously

distributed defects. The simple cases of 0-dimensional material structures and $n$-dimensional material structures are considered in Section 6 . Sections 7 and 8 present the physically relevant cases of dislocations and disclinations, and some examples are given. Thus, all the cases relevant to the 3dimensional space are covered. Section 9 considers the motion of material structure and the associated defects, and the rate of change of the motion. Both the continuous and singular cases are discussed for the case where the material structure is carried with a family of diffeomorphism of the body manifold. Finally, we give an example in which a smooth distribution of defects evolves into a singular defect.

\section{FORMS AND HYPERSURFACES}

Defects are considered in this article to be obstacles to integrability. Consider a family $\mathcal{F}$ of $(n-r)$-dimensional oriented hypersurfaces in the body manifold $\mathcal{M}$. We view the family $\mathcal{F}$ as a given material structure in the 
body. For example, a family of 2-dimensional surfaces in a 3-dimensional body may be thought of as a family of lattice layers. Let $\mathcal{S}$ be an $(r+$ 1)-dimensional submanifold with boundary in the body manifold. The "amount" hypersurfaces belonging to $\mathcal{F}$ that cross the boundary $\partial \mathcal{S}$, if different from zero, indicates the generation or annihilation of such hypersurfaces in $\mathcal{S}$. We view such creation or annihilation of material hypersurfaces as an indication for the presence of defects in $\mathcal{S}$.

In this section, we describe the notions from exterior calculus used for the description of what is referred to above as a "family of hypersurfaces" in the body manifold $\mathcal{M}$.

We recall [Ste83, pp. 16-17] that an $r$-dimensional subspace $W$ of a vector space $V$ is associated with a decomposable $r$-vector $\mathfrak{v}$ which is unique up to a scalar factor such that $u \in W$ if and only if $\mathfrak{v} \wedge u=0$. In the sequel we will use this property for subspaces $D_{x}^{*}$ of the various cotangent spaces $T_{x}^{*} \mathcal{M}, x \in \mathcal{M}$ of dimension $p=n-r$. It is observed that each $D_{x}^{*}$ determines a unique $r$-dimensional subspace $D_{x}=\left(D_{x}^{*}\right)^{\perp}=\left\{v \in T_{x} \mathcal{M} \mid\right.$ $\omega(v)=0$, for all $\left.\omega \in D_{x}^{*}\right\}$. Thus, a $p$-dimensional subspace $D_{x}^{*} \subset T_{x}^{*} \mathcal{M}$ is determined by a decomposable $p$-covector (alternating tensor) $\varphi$.

We will use the notation $v\lrcorner \omega$ for the contraction of a $p$-covector $\omega$ with a vector $v$, a $(p-1)$-covector satisfying

$$
v\lrcorner \omega\left(w_{1}, \ldots, w_{p-1}\right)=\omega\left(v, w_{1}, \ldots, w_{p-1}\right)=\omega\left(v \wedge w_{1} \wedge \cdots \wedge w_{p-1}\right) .
$$

Let $\omega$ be a $q$-covector, $q<p$, such that $\varphi \wedge \omega=0$. Then, recalling the identity

$$
\left.v\lrcorner(\varphi \wedge \omega)=(v\lrcorner \varphi) \wedge \omega+(-1)^{r} \varphi \wedge(v\lrcorner \omega\right),
$$

for any tangent vector $v$, one has,

$$
\varphi \wedge(v\lrcorner \omega)=0
$$

for every vector $v$ that annihilates $\varphi$ in the sense that $v\lrcorner \varphi=0$. In the particular case $q=1$, and assuming $\varphi \neq 0$, one has $\omega(v)=0$.

Conversely, if $\omega(v)=0$ for every $v \in D_{x}$,

$$
(v\lrcorner \varphi) \wedge \omega=0
$$

for every covector $\omega$ such that $\varphi \wedge \omega=0$. Since $\varphi$ is decomposable, there is a basis $\left\{\varphi^{1}, \ldots, \varphi^{n}\right\}$ of $T_{x} \mathcal{M}$ such that $\varphi$ can be expressed as $\varphi=\varphi^{1} \wedge \cdots \wedge$ $\varphi^{p}$. Hence,

$$
v\lrcorner \varphi=\sum_{i=1}^{p}(-1)^{i-1} v^{i} \varphi^{1} \wedge \cdots \wedge \widehat{\varphi}^{i} \wedge \cdots \wedge \varphi^{p} .
$$

For any $j=1, \ldots, p, \varphi \wedge \varphi^{j}=0$, hence,

$$
0=(v\lrcorner \varphi) \wedge \varphi^{j}=(-1)^{p-j-1} v^{j} \varphi^{1} \wedge \cdots \wedge \varphi^{p} .
$$

It follows that $v^{j}=0$, for all $j=1, \ldots, p$, so that $\left.v\right\lrcorner \varphi=0$. We conclude that $v \in D_{x}$ if and only if

and $\operatorname{dim} D_{x}=r=n-p$.

$$
v\lrcorner \varphi=0
$$


Remark 1. Clearly, in a dual procedure and as given in [Ste83, pp. 16-17], one could start with a simple $r$-vector $\mathfrak{v}$ at a point $x \in \mathcal{M}$ and define the subspace $D_{x}=\left\{v \in T_{x} \mathcal{M} \mid \mathfrak{v} \wedge v=0\right\}$. Then, the orthogonal subspace is given by $D_{x}^{*}=\left\{\alpha \in T_{x}^{*} \mathcal{M} \mid \alpha(v)=0, v \in D_{x}\right\}$. For an $r$-vector $\mathfrak{v}$ and a $k$-covector $\omega$, with $r \geqslant k$, we use the inner product notation $\mathfrak{v}\llcorner\omega$, an $(r-k)$-vector defined by,

$$
\varphi(\mathfrak{v}\llcorner\omega)=(\varphi \wedge \omega)(\mathfrak{v}),
$$

for every $(r-k)$-covector $\varphi$. The condition that $\alpha \in D_{x}^{*}$ may then be written as

$$
\mathfrak{v}\llcorner\alpha=0 .
$$

A smooth decomposable differential $p$-form $\varphi$ will induce therefore a distribution $D$ on $\mathcal{M}$ of dimension $r=n-p$. Here, by a "distribution" we mean a subbundle of the tangent bundle rather than a Schwartz distribution. Conversely, a distribution $D$ of dimension $r=n-p$ will induce a collection of forms such that if $\varphi$ induces $D$, so would the form $a \varphi$ for any positive, real valued function $a$ on $\mathcal{M}$.

Let $v_{1}, \ldots, v_{p} \in T_{x} \mathcal{M}$. We interpret $\varphi(x)\left(v_{1}, \ldots, v_{p}\right)$ as the amount of hyperplanes belonging to the distribution that cross the infinitesimal $p$ dimensional oriented element (a $p$-dimensional parallelepiped or a simplex) generated by the vectors $v_{1}, \ldots, v_{p}$. In particular, if for some $i=$ $1, \ldots, p, v_{i} \in D_{x}$, so that $\left.v\right\lrcorner \varphi(x)=0$, this quantity will vanish as the hyperplanes and the subspace generated by $v_{1}, \ldots, v_{p}$ intersect on a subspace of dimension greater than zero. Multiplying the form $\varphi$ by a positive function $a$, the resulting form $a \varphi$ is interpreted as describing a family of hyperplanes which are parallel to those represented by $\varphi$, and whose density a times larger.

A distribution does not represent necessarily tangent spaces to a family of hypersurfaces, as we wish to consider. It is recalled that an $r$-dimensional submanifold $\mathcal{S}$ is an integral manifold of the distribution if $T_{x} \mathcal{S}=D_{x}$ for all $x \in \mathcal{S}$. A distribution $D$ is referred to as involutive if at each $x \in \mathcal{M}$, $D_{x}$ is the tangent space of an $r$-dimensional integral manifold. The Frobenius theorem implies (e.g., AMR88, pp. 441-442]) that the distribution $D$ is involutive if and only if there is a 1 -form $\beta$ on $\mathcal{M}$ such that

$$
\mathrm{d} \varphi=\beta \wedge \varphi \text {. }
$$

Consider the form $\varphi_{a}=a \varphi$ for a function $a$. Recalling the identity

$$
\mathrm{d}(\mu \wedge v)=\mathrm{d} \mu \wedge v+(-1)^{q} \mu \wedge \mathrm{d} v,
$$

for the $q$-form $\mu$ and a form $v$ over $\mathcal{M}$, one has

$$
\mathrm{d} \varphi_{a}=\mathrm{d} a \wedge \varphi+a \mathrm{~d} \varphi .
$$

Assume that condition (2.10) holds. Then, if the function $a$ is a solution of the equation $\mathrm{d} a=-a \beta,(2.12)$ implies that $\mathrm{d} \varphi_{a}=0$. Conversely, assume that $\mathrm{d} \varphi_{a}=0$ for some positive function $a$. Then, the one form $\beta=-\mathrm{d} a / a$ 
satisfies the integrability condition (2.10). We conclude therefore that the distribution induced by a form $\varphi$ is involutive if and only if it has an integrating factor, a function $a$ on $\mathcal{M}$ such that $\mathrm{d}(a \varphi)=0$. Thus, for a form that induces an involutive distribution, the density of the hyperplanes at each point may be readjusted so that the exterior derivative of the resulting form vanishes. In particular, if $\mathrm{d} \varphi=0$, the distribution induced by $\varphi$ is involutive.

Remark 2. Let $D$ be a distribution induced by a simple $r$-vector field $\mathfrak{v}$ represented locally by $v_{1} \wedge \cdots \wedge v_{r}$ for smooth vector fields $v_{1}, \ldots, v_{r}$. Then, using the Lie bracket notation, the condition that the distribution is involutive is that $\left[v_{i}, v_{j}\right]$ is also a section of $D$ for all $i, j=1, \ldots, r$. It is noted however that we did not write a condition yet on $\mathfrak{v}$ that will be equivalent to the condition $\mathrm{d} \varphi=0$. The theory of de Rham currents provides one with the required tools for writing such a condition.

\section{STRUCTURE Forms, DEFECT FormS AND THE CORRESPONDING FRANK'S RULE}

From the point of view of the material structure of bodies, any decomposable $p$-form represents a distribution of hyperplanes, Bravais hyperplanes, at the various points in the body while an involutive distribution represents a collection of submanifolds at the various material points i.e., hyperplanes at various points may be assembled to form tangent spaces of $n-p=r$-dimensional submanifolds-the material or Bravais hypersurfaces. We will refer to such decomposable forms as structure forms.

The material structure described by an involutive structure $p$-form may still contain defects. Such defects are due to the creation or loss of material hypersurfaces in some regions in the body. Let $\mathcal{S}$ be an $(r+1)$-dimensional manifold with a boundary. The creation or loss of material hypersurfaces inside $\mathcal{S}$ will be reflected by the integral of the structure form over the boundary, $\partial \mathcal{S}$. Note that the integrals of a form over $\mathcal{S}$ and its boundary make sense even if the form is not involutive. In this case, the integral over the boundary may naturally be interpreted as the creation of hyperplanes rather than hypersurfaces.

Stokes's theorem asserts that

$$
\int_{\partial \mathcal{S}} \varphi=\int_{\mathcal{S}} \mathrm{d} \varphi
$$

Thus, if the exterior derivative $\mathrm{d} \varphi$ of the structure form vanishes, the total creation or annihilation of material hypersurfaces within any $(r+1)$ submanifold $\mathcal{S}$, as reflected in the total amount of hypersurfaces that cross the boundary $\partial \mathcal{S}$, will vanish. In other words, for a decomposable, form $\varphi$ satisfying (2.10), which, by the Frobenius theorem, induces a family of hypersurfaces, the stronger condition, $\mathrm{d} \varphi=0$, i.e., $\varphi$ is closed, implies that the family of hypersurfaces have no sources or sinks. This suggests that $\mathrm{d} \varphi$

is the measure of the sources of material, or Bravais, hypersurfaces inside 
the body $\mathcal{M}$-the measure of the distribution of defects. We will refer to $\mathrm{d} \varphi$ as the defect form corresponding to $\varphi$.

It is recalled that the skew symmetry of the exterior derivative combined with the symmetry of second derivatives of functions implies that for any form $\alpha$,

$$
\mathrm{d}^{2} \alpha:=\mathrm{d}(\mathrm{d} \alpha)=0 .
$$

Let $\psi=\mathrm{d} \varphi$ be the defect form associated with the structure form $\varphi$. It follows, therefore, that $\psi$ must satisfy the condition

$$
\mathrm{d} \psi=0 .
$$

This compatibility condition is the analog of Frank's rules for defects of any dimension on manifolds, as long they are smoothly distributed.

\section{De Rham Currents}

Let $\varphi$ be a decomposable $p$-covector at a point $x \in \mathcal{M}$. It follows that one may choose a basis $\left\{\boldsymbol{e}_{i}\right\}, i=1, \ldots, n$, of $T_{x} \mathcal{M}$ with dual basis $\left\{\varphi^{i}\right\}$ such that $\varphi=\varphi^{1} \wedge \cdots \wedge \varphi^{p}$. Let $\omega$ be an $(n-p)$-covector such that $\varphi \wedge \omega \neq 0$. Then, $\omega$ must be of the form $\omega=a \varphi^{p+1} \wedge \cdots \wedge \varphi^{n}+\alpha$, with $\varphi \wedge \alpha=0$, for some nonvanishing number $a$. The subspaces induced by $\varphi$ are spanned by $\left\{\boldsymbol{e}_{p+1}, \ldots, \boldsymbol{e}_{n}\right\}$. Let $\left\{v_{1}, \ldots, v_{n}\right\}$ be $n$ vectors in $T_{x} \mathcal{M}$ and consider $\varphi \wedge$ $\omega\left(v_{1}, \ldots, v_{n}\right)=\varphi \wedge \omega\left(v_{1} \wedge \cdots \wedge v_{n}\right)$. Then, $v_{1} \wedge \cdots \wedge v_{n}$ must be of the form

$$
v_{1} \wedge \cdots \wedge v_{n}=b \boldsymbol{e}_{1} \wedge \cdots \wedge \boldsymbol{e}_{n}
$$

for some real number $b$. The orthogonality of the two forms with vectors in the respective subspaces implies that

$$
\varphi \wedge \omega\left(v_{1} \wedge \cdots \wedge v_{n}\right)=\varphi\left(\boldsymbol{e}_{1}, \ldots, \boldsymbol{e}_{p}\right) \omega\left(b \boldsymbol{e}_{p+1} \wedge \cdots \wedge \boldsymbol{e}_{n}\right)=a b .
$$

This quantity, as well as the identical $\left(\left(v_{1} \wedge \cdots \wedge v_{n}\right)\llcorner\varphi)(\omega)\right.$, is interpreted as the amount of cells formed by the hyperplanes induced by the forms $\varphi$ and $\omega$ contained in the $n$-parallelepiped determined by $v_{1}, \ldots, v_{n}$.

Accordingly, for a $p$-form $\varphi$ and an $(n-p)$-form $\omega$, one may interpret the integral

$$
\int_{\mathcal{M}} \varphi \wedge \omega
$$

as the total amount of cells in $\mathcal{M}$.

Therefore, one may consider the linear operator $T_{\varphi}$ acting on $(n-p)$ forms by

$$
T_{\varphi}(\omega)=\int_{\mathcal{M}} \varphi \wedge \omega
$$

whose action on an $(n-p)$-form $\omega$ gives the total amount of cells corresponding to $\varphi \wedge \omega$ in $\mathcal{M}$.

A linear functional $T_{\varphi}$ acting on differential forms as in (4.4) is a typical simple example of a de Rham current. 
A de Rham $r$-current is a linear operator acting on the space of smooth $r$ forms with compact supports. A de Rham current $T$ is required to be continuous in the following sense. Let $\left(\omega_{k}\right)$ is a sequence of $r$-forms whose supports are all contained in a compact subset of a coordinate neighborhood and whose local representatives as well as all the partial derivatives of all orders of the local representatives tend to zero uniformly as $k \rightarrow \infty$. Then, $T\left(\omega_{k}\right) \rightarrow 0$. Thus, for the case $r=0, T$ is a Schwartz distribution on the manifold $\mathcal{M}$. For $r>0$, currents contain additional geometric properties in comparison with Schwartz distributions.

In contrast with the example above where the $r$-current $T_{\varphi}$ was induced by a smooth $(n-r)$-form $\varphi$, currents may exhibit singular behavior. As a typical simple example, an $r$-dimensional submanifold $\mathcal{S} \subset \mathcal{M}$ induces a current $T_{\mathcal{S}}$ defined by

$$
T_{\mathcal{S}}(\omega)=\int_{\mathcal{S}} \omega
$$

for every $r$-form $\omega$ with compact support. In comparison with (4.4), the current $T_{\mathcal{S}}$ may be viewed as the limit of currents of the form $T_{\varphi}$ where the support of $\varphi$ shrinks to a small neighborhood of $\mathcal{S}$ and the value of its components tend to infinity in that neighborhood. This process may be made rigorous by the process of regularization (e.g., [dR84, pp. 61-70]) which is a generalization of the analogous process for Schwartz distributions.

A current may be restricted to the domain of a chart on $\mathcal{M}$ by restricting its action to forms supported in the domain of that chart. An $r$-form $\omega$ which is supported in the domain of a chart, may be expressed using real valued functions $\omega_{\mu}$ as

$$
\omega=\sum_{(\mu)} \omega_{\mu} \mathrm{d} x^{\mu}
$$

where $\mu$ is an increasing (indicated by the parenthesis around it) $r$-multiindex taking values in the range $1, \ldots, n$. By linearity,

$$
T(\omega)=\sum_{(\mu)} T\left(\omega_{\mu} \mathrm{d} x^{\mu}\right)=\sum_{(\mu)} T^{\mu}\left(\omega_{\mu}\right),
$$

where $T^{\mu}$ are the Schwartz distributions, 0-currents, so that $T^{\mu}\left(\omega_{\mu}\right)=$ $T\left(\omega_{\mu} \mathrm{d} x^{\mu}\right)$. For an $m$-vector field $\mathfrak{v}$ and an $r$-current $T$, consider the $(r+m)$ current $T \wedge \mathfrak{v}$ defined by

$$
(T \wedge \mathfrak{v})(\omega)=T(\mathfrak{v}\lrcorner \omega) .
$$

Here, in analogy with (2.1) $\mathfrak{v}\lrcorner \omega$ is the $r$-form such that for any $r$-vector field $\mathfrak{w}$,

$$
\mathfrak{v}\lrcorner \omega(\mathfrak{w})=\omega(\mathfrak{v} \wedge \mathfrak{w}) .
$$

Then, the restriction of a current $T$ to a chart with domain $U \subset \mathcal{M}$ may be represented locally by distributions $T^{\lambda}$ in the form

$$
\left.T\right|_{U}=\sum_{(\lambda)} T^{\lambda} \wedge \frac{\partial}{\partial x^{\lambda}} .
$$


This representation views a current as a generalized multivector field which is the approach of [Whi57, p. 199]. Using a partition of unity, a current may be represented by its restrictions to the domains of charts.

For a smooth $m$-form $\alpha$ and an $r$-current $T$ with $r>m$, the $(r-m)$ current $T\llcorner\alpha$ is defined by

$$
T\llcorner\alpha(\omega)=T(\alpha \wedge \omega) .
$$

Using this notation, de Rham's representation of currents may be expressed as follows. Let $\left\{T_{\mu}\right\}$, where $\mu$ is an increasing multi-index with $\mu_{i}=1, \ldots, n$, $i=1, \ldots, n-r$, be $n$-currents in a coordinate neighborhood. Consider the $r$-current

$$
T=\sum_{(\mu)} T_{\mu}\left\llcorner\mathrm{d} x^{\mu}\right.
$$

so that

$$
T(\omega)=\sum_{(\mu)} T_{\mu}\left(\mathrm{d} x^{\mu} \wedge \omega\right)
$$

Using the linearity of the currents, it may be shown that the restriction of a current to a coordinate neighborhood may be represented in the form (4.12). It is noted that in (4.12), currents are viewed as generalized forms.

Let $T$ be a current which is given in a coordinate neighborhood in $\mathcal{M}$ by the single Schwartz distribution $T^{0}$ and an $r$-vector field $\mathfrak{v}$ in the form

$$
T=T^{0} \wedge \mathfrak{v}
$$

Then, $T\llcorner\alpha=0$ for every 1-form $\alpha$ that takes values in the distribution $D^{*}$ which is orthogonal to that induced by $\mathfrak{v}$. Conversely, let $D^{*}$ be a $p$ dimensional subbundle of $T^{*} \mathcal{M}$ and assume that for a current $T, T\llcorner\alpha=0$ for every 1 -form $\alpha$ valued in $D^{*}$. Then, $T$ is of the form (4.14) where $\mathfrak{v}$ is a multivector that induces the distribution $D$ which is orthogonal to $D^{*}$.

The boundary of an $r$-current $T$ is the $(r-1)$-current $\partial T$ defined by the condition

$$
\partial T(\omega)=T(\mathrm{~d} \omega)
$$

Consider the $r$-current $T_{\varphi}$ defined in terms of a smooth $(n-r)$-form $\varphi$ as in (4.4). Then, using (2.11), Stokes's theorem and the fact that $\omega$ has a compact support in $\mathcal{M}$, one has

$$
\begin{aligned}
\partial \mathrm{T}_{\varphi}(\omega) & =\int_{\mathcal{M}} \varphi \wedge \mathrm{d} \omega, \\
& =(-1)^{n-r}\left[\int_{\mathcal{M}} \mathrm{d}(\varphi \wedge \omega)-\int_{\mathcal{M}} \mathrm{d} \varphi \wedge \omega\right], \\
& =(-1)^{n-r}\left[\int_{\partial \mathcal{M}} \varphi \wedge \omega-\int_{\mathcal{M}} \mathrm{d} \varphi \wedge \omega\right], \\
& =(-1)^{n-r+1} \int_{\mathcal{M}} \mathrm{d} \varphi \wedge \omega .
\end{aligned}
$$


It follows that,

$$
\partial T_{\varphi}=(-1)^{n-r+1} T_{\mathrm{d} \varphi}
$$

For an $r$-dimensional submanifold with boundary $\mathcal{S}$, the boundary of the current $T_{\mathcal{S}}$ defined in (4.5) satisfies

$$
\begin{aligned}
\partial T_{\mathcal{S}}(\omega) & =T_{\mathcal{S}}(\mathrm{d} \omega), \\
& =\int_{\mathcal{S}} \mathrm{d} \omega \\
& =\int_{\partial \mathcal{S}} \omega .
\end{aligned}
$$

Hence,

$$
\partial T_{\mathcal{S}}=T_{\partial \mathcal{S}}
$$

which motivates the terminology used.

Finally, since for every form $\omega, \mathrm{d}^{2} \omega=0$, one has $\partial^{2} T(\omega)=\partial(\partial T(\omega))=$ $T\left(\mathrm{~d}^{2} \omega\right)$, and we conclude that

$$
\partial^{2} T=0
$$

identically.

\section{Structure Currents, Defect CurRents And Frank's Rules}

It is concluded from the previous section that de Rham currents may be thought of as generalizations of smooth differential forms to the singular, non-smooth, case, or alternatively, as generalization of smooth multivector fields to the singular case. In addition, the boundary of a current generalizes the exterior derivative of a form. Thus, an $r$-current will be the singular counterpart of a $p=n-r$ structure form and will be referred to as a structure current. Accordingly, for a structure current $T$, the boundary $\partial T$ will represent the geometry of the defects and will be referred to as the defect current. The material structure represented by the current $T$ will be defect free if $\partial T=0$.

We recall that the constancy theorem for currents asserts that on a connected manifold $\mathcal{M}$, a closed $n$-current $T$, i.e., $T$ satisfies $\partial T=0$, is represented by a constant $c$ in the form

$$
T(\omega)=c \int_{\mathcal{M}} \omega
$$


One may apply this to the de Rham representation of currents (4.12) as follows. We observe first that by (4.12), one has

$$
\begin{aligned}
\partial T(\omega) & =T(\mathrm{~d} \omega)=\sum_{(\mu)} T_{\mu}\left(\mathrm{d} x^{\mu} \wedge \mathrm{d} \omega\right), \\
& =(-1)^{n-r} \sum_{(\mu)} T_{\mu}\left(\mathrm{d}\left(\mathrm{d} x^{\mu} \wedge \omega\right)\right), \\
& =(-1)^{n-r} \sum_{(\mu)} \partial T_{\mu}\left(\mathrm{d} x^{\mu} \wedge \omega\right), \\
& =(-1)^{n-r} \sum_{(\mu)} \partial T_{\mu}\left\llcorner\mathrm{d} x^{\mu}(\omega),\right.
\end{aligned}
$$

where (2.11) was used in the second line. It follows that the boundary of the current $T$ can always be represented by the $(n-1)$-currents $\partial T_{\mu}$ in the form

$$
\partial T=(-1)^{n-r} \sum_{(\mu)} \partial T_{\mu}\left\llcorner\mathrm{d} x^{\mu} .\right.
$$

Assume that the current $T$ is given the form

$$
T=T_{0}\left\llcorner\varphi=T_{0}\left\llcorner\left(\varphi^{1} \wedge \cdots \wedge \varphi^{n-r}\right)\right.\right.
$$

for an $n$-current $T_{0}$ and a collection of $n-r$ linearly independent1-forms $\varphi^{i}$, $i=1, \ldots, n-r$ spanning a subbundle $D^{*}$ of $T^{*} \mathcal{M}$. The current $T$ can thus be associated with the distribution $D^{*}$. In particular, let $\psi$ be any 1 -form valued in $D^{*}$, then, for each $(r-1)$-form $\omega$,

$$
\begin{aligned}
(T\llcorner\psi)(\omega) & =\left(T_{0}\llcorner\varphi)(\psi \wedge \omega),\right. \\
& =T_{0}(\varphi \wedge \psi \wedge \omega), \\
& =0 .
\end{aligned}
$$

Thus, $T\llcorner\psi=0$.

Conversely, assume that for a general current $T$, we are given that $T\llcorner\psi=$ 0 for every section $\psi$ of a subbundle $D^{*}$. We consider the restriction of $T$ to a coordinate neighborhood in which $D^{*}$ is induced by the form $\varphi=$ $\varphi^{1} \wedge \cdots \wedge \varphi^{n-r}$ in which $\varphi^{1}, \ldots, \varphi^{n}$ span $T^{*} \mathcal{M}$. Writing $T=\sum_{(\lambda)} T_{\lambda}\left\llcorner\varphi^{\lambda}\right.$, it follows that for any $(r-1)$-form $\omega$ and all sections $\psi$ of $D^{*}$,

$$
\begin{aligned}
0 & =(T\llcorner\psi)(\omega), \\
& =\sum_{(\lambda)}\left(\left(T_{\lambda}\left\llcorner\varphi^{\lambda}\right)\llcorner\psi)(\omega),\right.\right. \\
& =\sum_{(\lambda)} T_{\lambda}\left(\varphi^{\lambda} \wedge \psi \wedge \omega\right) .
\end{aligned}
$$

Since $\varphi^{1 \ldots n-r} \wedge \psi=0$, it follows that $T_{\lambda}=0$ for all $\lambda \neq 1, \ldots, r$. Hence, $T=T_{1 \ldots n-r}\left\llcorner\left(\varphi^{1} \wedge \cdots \wedge \varphi^{n-r}\right)\right.$. We conclude that $T\llcorner\psi=0$ for every section of a subbundle $D^{*} \subset T^{*} \mathcal{M}$, if an only if

$$
T=T_{0}\llcorner\varphi
$$


for an $n$-current $T_{0}$ and an $(n-r)$-form $\varphi$ associated with $D^{*}$. We will refer to such a current as a decomposable current. It is observed that the condition $T\left\llcorner\psi=0\right.$ for every section $\psi$ of $D^{*}$, induces an ideal on the collection of forms in the sense that for each $q$-form $\alpha$, with $q \leqslant n-r-1, T\llcorner\psi=0$ for all $\psi$ implies that $T\llcorner(\psi \wedge \alpha)=(T\llcorner\psi) \wedge \alpha=0$ also.

Finally, if $S$ is a current representing the defect structure, the identity $\partial^{2} T=0$ implies that $\partial S=0$, necessarily. This is the generalization of Frank's rules for a possibly singular defect structure.

\section{The Simple CASES}

In this section we consider the simple, possibly trivial, cases of $n$-currents and 0-currents, where it is recalled that 0-currents are Schwartz distributions on the manifold $\mathcal{M}$.

6.1. 0-Forms, $n$-Currents and Nonuniformity. A 0 -form $\varphi$ on $\mathcal{M}$ is a real valued differentiable function. One may interpret the form $\varphi$ as a field describing a certain intensive property in $\mathcal{M}$ such as the temperature field, a certain potential field, etc. A 0 -form does not induce nontrivial hyperplanes and so no real material structure is represented by $\varphi$. In addition, the condition $\mathrm{d} \varphi=0$ is not really a condition of integrability as $\varphi$ cannot be the exterior derivative of a form. However, the nonuniformity of $\varphi$, implied by $\mathrm{d} \varphi \neq 0$ may still be regarded as a representation of a field of defects. This is manifested clearer in the case where we consider currents. The currents under consideration will be of order $n$.

Consider for example an $n$-dimensional submanifold with boundary $\mathcal{B} \subset$ $\mathcal{M}$. Let $T_{\mathcal{B}}$ be the $n$-current in $\mathcal{M}$ given by

$$
T_{\mathcal{B}}(\omega)=\int_{\mathcal{B}} \omega
$$

It follows from (4.19) that $\partial T_{\mathcal{B}}(\alpha)=T_{\partial \mathcal{B}}(\alpha)$. This identity suggests that the boundaries of bodies be interpreted as defects. The condition $\partial^{2} T_{\mathcal{B}}=0$ simply imply in this case that the boundary of $\partial \mathcal{B}$ vanishes.

6.2. Schwartz Distributions: The Case of 0-Currents. Differential forms of degree $n$ may be integrated over bounded subsets of the material manifold $\mathcal{M}$. As such, from the physical point of view, they represent densities of extensive properties such as the mass density or electric charge density. Such forms may be paired with smooth 0 -forms of compact supports, that is, with test functions over $\mathcal{M}$. Thus, if $\rho$ is an $n$-form, one may consider the 0 -current $T_{\rho}$ given by,

$$
T_{\rho}(\varphi)=\int_{\mathcal{M}} \rho \varphi
$$

for every test function $\varphi$. Evidently, the product with the test function $\varphi$ cuts off the integrand so that if $\rho$ is measurable, the integral is well defined. 
The test function $\varphi$ may be interpreted as a potential so that $\varphi \rho$ may be interpreted as the corresponding energy density.

An $(n-1)$-form $\sigma$ induces another construction of a 0 -current $\partial T_{\sigma}$ by

$$
\partial T_{\sigma}(\varphi)=T_{\sigma}(\mathrm{d} \varphi)=\int_{\mathcal{M}} \sigma \wedge \mathrm{d} \varphi
$$

It is noted that $T_{\sigma}$ is a 1-current induced by the form $\sigma$. If we interpret the test form $\varphi$ as a potential, $\mathrm{d} \varphi$ may be interpreted as (minus) the corresponding force field and $\sigma$ may be interpreted as the flux field for some extensive property under consideration, so that $\sigma \wedge \mathrm{d} \varphi$ is the density of power.

For any $n$-form $\rho$, one has $\mathrm{d} \rho=0$. In analogy, 0 -currents have no boundary. Hence, no defects may be associated with such densities. Nevertheless, we may interpret the $n$-form $\rho$ as the void fraction or density of vacancies in the body.

Singular 0-currents are singular distributions defined on the manifold $\mathcal{M}$. Thus, in addition to currents induced by $n$-forms as in (6.2), one may consider distributions such as the Dirac measure $\delta_{x}$ at a point $x \in \mathcal{M}$, i.e., the current defined by

$$
\delta_{x}(\varphi)=\varphi(x)
$$

for any test function $\varphi$. In addition, for a 0 -current $T$ and a vector field $w$, one may consider the 0 -current $\partial(w \wedge T)$ which acts on test functions by

$$
\partial(w \wedge T)(\varphi)=(w \wedge T)(\mathrm{d} \varphi)=T(\mathrm{~d} \varphi(w)) .
$$

For example,

$$
\partial\left(w \wedge \delta_{x}\right)(\varphi)=\delta_{x}(\mathrm{~d} \varphi(w))=(\mathrm{d} \varphi(x))(w(x))
$$

which is the directional derivative of $\varphi$ at $x$ in the direction of $w(x)$.

Singular 0-currents may be interpreted as concentrated vacancies or inclusions. For example, $(\mathrm{d} \varphi(x))(w(x))$ may be interpreted as the power expended by the force $\mathrm{d} \varphi(x)$ for the velocity $w(x)$ of the concentrated inclusion.

\section{Dislocations}

The description of smooth distributions of dislocations in terms of differential forms on general manifolds and the generalization to singular dislocations using de Rham currents are discussed in our previous paper [ES12]. Here, following the general introduction below and the reviewing the example of an edge dislocation, we will consider the example of a screw dislocation which we did not consider in [ES12]. Finally, we will demonstrate how the Frank rules follow from the condition $\partial^{2} T=0$.

7.1. The Geometry of Dislocations. Continuous distributions of dislocations in the body $\mathcal{M}$ are associated with the integrability issue of a 1 -form $\varphi$. Each 1-form is trivially decomposable and as such, it induces at each $x \in \mathcal{M}$ a hyperplane $D_{x}$ which we interpret as the Bravais hyperplane at that point. Multiplying $\varphi$ be a positive function $a$ will have the effect of 
changing the density of the Bravais hyperplanes. In fact, the covector $\varphi(x)$ is intimately related to the Miller indices for the Bravais planes at $x$. It is natural therefore to refer to the 1-form $\varphi$ as the layering form.

Rather than considering the Burgers vector obtained by tracing a loop in the non dislocated body and evaluating the vector needed to close the loop in the dislocated state, we envisage an integration over a closed loop of the form $\varphi$ which is interpreted as the total amount of hyperplanes that penetrate the loop in one particular orientation. It is noted that being a 1-form, the distribution induced by $\varphi$ is not necessarily involutive. For a smooth layering 1-form $\varphi$, the distribution of dislocations is modeled by $\mathrm{d} \varphi$. In case $\mathrm{d} \varphi=0$, locally, by the Poincare lemma there is a function $u$ such that $w=\mathrm{d} u$. We view $u$ as a labeling function for the Bravais hypersurfaces. For additional examples to those given below, see [ES12].

For the singular case, the layering is modeled by an $(n-1)$-structure current $T$ and the dislocations are described by its boundary $\partial T$.

7.2. Edge Dislocations. Assume that $\mathcal{M}$ is an $n$-dimensional manifold without boundary and let $\mathcal{S}$ be an $(n-1)$-submanifold with boundary of $\mathcal{M}$. We consider the $(n-1)$-structure current $T_{\mathcal{S}}$ given by (4.5). Then, as shown in (4.19), the dislocation $(n-2)$-current is given by $T_{\partial \mathcal{S}}$.

As a concrete example in $\mathbb{R}^{3}$, consider the case where $\mathcal{M}=(-1,1)^{3}$ is an open cube in $\mathbb{R}^{3}$. Let

$$
\mathcal{S}=\left\{\left(0, x^{2}, x^{3}\right) \in \mathcal{M} \mid x^{2} \leqslant 0\right\}
$$

equipped with the orientation induced by the form $\mathrm{d} x^{2} \wedge \mathrm{d} x^{3}$. The current $T_{\mathcal{S}}$ represents an added half plane. Then, $\partial T_{\mathcal{S}}=T_{\partial \mathcal{S}}$, where $\partial \mathcal{S}=$ $\left\{\left(0,0, x^{3}\right) \in \mathcal{M}\right\}$, oriented naturally by the form $\mathrm{d} x^{3}$, is the singular dislocation line as expected.

7.3. Screw Dislocations. We present here an additional example, that of a screw dislocation.

Let $L \subset \mathbb{R}^{3}$ be given by $L=\{(0,0\} \times \mathbb{R}\}=\{(0,0, z) \mid z \in \mathbb{R}\}$ and let $D \subset \mathbb{R}^{3}$ be given by $D=\mathbb{R}^{3} \backslash L=\left\{(x, y, z) \in \mathbb{R}^{3} \mid(x, y) \neq(0,0)\right\}$. It is noted that on $D$ we may use a cylindrical coordinate system $(r, \theta, z)$, where we take the domain $[0,2 \pi)$ for $\theta$ without using a proper atlas on the unit circle.

Consider the layering 1-form $\varphi$ on $D$ defined by

$$
\varphi=-\frac{b}{2 \pi} \mathrm{d} \theta+\mathrm{d} z \text {. }
$$

Evidently, as its components are constants, $\varphi$ is a closed form. It thus follows from Poincare's lemma that locally $\varphi$ is exact. Since $D$ is not contractible to a point, $\varphi$ is not exact globally. In fact, in the open set $D \backslash$ $\{(r, \theta, z) \mid \theta=0\}, \varphi=\mathrm{d} F$ for the real valued

$$
F(r, \theta, z)=-\frac{b \theta}{2 \pi}+z
$$


whose level sets

$$
z=\frac{b \theta}{2 \pi}+C, \quad C \in \mathbb{R}
$$

describe spiraling screw threads of pitch $b$.

For any $r>0$, let $S_{r, l}=\left\{(x, y, z) \in \mathbb{R}^{3} \mid x^{2}+y^{2}=r^{2}, z=l\right\}$ be the circle of radius $r$ situated at $z=l$ and let $\iota: S_{r, l} \rightarrow D$ be the inclusion. Then, for example,

$$
\begin{aligned}
\int_{S_{r, l}} \varphi & =\int_{S_{r, l}} \iota^{*}(\varphi), \\
& =\int_{S_{r, l}}-\frac{b}{2 \pi} \mathrm{d} \theta, \\
& =-b .
\end{aligned}
$$

(It is observed that $\iota^{*}(\varphi)(\partial / \partial \theta)=\varphi\left(\iota_{*}(\partial / \partial \theta)\right)=\varphi(\partial / \partial \theta)=-b / 2 \pi$.)

We now consider the structure 2 -current $T_{\varphi}$ in $\mathbb{R}^{3}$, given by

$$
T_{\varphi}(\omega)=\int_{D} \varphi \wedge \omega
$$

for any 2-form $\omega$ on $\mathbb{R}^{3}$ with compact support. In order to determine the associated geometry of the dislocation, we examine the defect current, the boundary $\partial T_{\varphi}$. For any 1 -form $\alpha$, we have,

$$
\begin{aligned}
\partial T_{\varphi}(\alpha) & =T_{\varphi}(\mathrm{d} \alpha), \\
& =\int_{D} \varphi \wedge \mathrm{d} \alpha, \\
& =-\int_{D} \mathrm{~d}(\varphi \wedge \alpha)+\int_{D} \mathrm{~d} \varphi \wedge \alpha .
\end{aligned}
$$

Since $\mathrm{d} \varphi=0$ in $D$, we conclude that

$$
\partial T_{\varphi}(\alpha)=-\int_{D} \mathrm{~d}(\varphi \wedge \alpha)
$$

Let $C_{\varepsilon}=\left\{(x, y, z,) \in \mathbb{R}^{3} \mid x^{2}+y^{2}<\varepsilon^{2}\right\}$ and let $D_{\varepsilon}=\mathbb{R}^{3} \backslash C_{\varepsilon}$. We may write

$$
\partial T_{\varphi}(\alpha)=-\int_{D} \mathrm{~d}(\varphi \wedge \alpha)=-\lim _{\varepsilon \rightarrow 0} \int_{D_{\varepsilon}} \mathrm{d}(\varphi \wedge \alpha) .
$$

Now it is noted that $D_{\varepsilon}$ is a manifold with a boundary. In fact, setting $S_{\varepsilon}=\left\{(x, y) \in \mathbb{R}^{2} \mid x^{2}+y^{2}=\varepsilon^{2}\right\}, \partial D_{\varepsilon}=S_{\varepsilon} \times \mathbb{R}$. We may therefore use Stokes's theorem in (7.9) and obtain

$$
\partial T_{\varphi}(\alpha)=-\lim _{\varepsilon \rightarrow 0} \int_{\partial D_{\varepsilon}} \iota^{*}(\varphi \wedge \alpha),
$$

where $\iota^{*}(\varphi \wedge \alpha)$ is the pullback under the inclusion $\iota: \partial D_{\varepsilon} \rightarrow D_{\varepsilon}$ which is simply the restriction of $\varphi \wedge \alpha$ to vectors tangent to $\partial D_{\varepsilon}$. 
A 1 -form $\alpha$ is represented by $\alpha=\alpha_{x} \mathrm{~d} x+\alpha_{y} \mathrm{~d} y+\alpha_{z} \mathrm{~d} z$ for the smooth functions $\alpha_{x}, \alpha_{y}$, and $\alpha_{z}$ defined on $\mathbb{R}^{3}$. In $D$, the form $\alpha$ may also be represented using cylindrical coordinates as $\alpha=\alpha_{r} \mathrm{~d} r+\alpha_{\theta} \mathrm{d} \theta+\alpha_{z} \mathrm{~d} z$. Since $\alpha_{x} \mathrm{~d} x+\alpha_{y} \mathrm{~d} y=\alpha_{r} \mathrm{~d} r+\alpha_{\theta} \mathrm{d} \theta$, using $x=r \cos \theta, y=r \sin \theta$ and

$$
\mathrm{d} x=\frac{\partial x}{\partial r} \mathrm{~d} r+\frac{\partial x}{\partial \theta} \mathrm{d} \theta, \quad \mathrm{d} y=\frac{\partial y}{\partial r} \mathrm{~d} r+\frac{\partial y}{\partial \theta} \mathrm{d} \theta
$$

one has

$$
\alpha_{\theta}=r\left(-\alpha_{x} \sin \theta+\alpha_{y} \cos \theta\right) .
$$

The restriction to $\partial D_{\varepsilon}$ satisfies

$$
\iota^{*}(\varphi \wedge \alpha)=\left(\varphi_{\theta} \alpha_{z}-\varphi_{z} \alpha_{\theta}\right) \mathrm{d} \theta \wedge \mathrm{d} z=\left(-\frac{b}{2 \pi} \alpha_{z}-\alpha_{\theta}\right) \mathrm{d} \theta \wedge \mathrm{d} z,
$$

and it follows that

$$
\begin{aligned}
\partial T_{\varphi}(\alpha) & =\lim _{\varepsilon \rightarrow 0} \int_{-\infty}^{\infty} \mathrm{d} z\left[\int_{S_{\varepsilon}}\left(\frac{b}{2 \pi} \alpha_{z}+\alpha_{\theta}\right) \mathrm{d} \theta\right], \\
& =\int_{-\infty}^{\infty} \mathrm{d} z\left\{\lim _{\varepsilon \rightarrow 0}\left[\int_{S_{\varepsilon}}\left(\frac{b}{2 \pi} \alpha_{z}-+\alpha_{\theta}\right) \mathrm{d} \theta\right]\right\} .
\end{aligned}
$$

Examining the limit in the second line of (7.14), we first note that

$$
\begin{aligned}
\lim _{\varepsilon \rightarrow 0} \int_{S_{\varepsilon}} \alpha_{\theta} \mathrm{d} \theta & =\lim _{\varepsilon \rightarrow 0} \int_{S_{\varepsilon}} \varepsilon\left(-\alpha_{x} \sin \theta+\alpha_{y} \cos \theta\right) \mathrm{d} \theta, \\
& =0,
\end{aligned}
$$

since $\alpha_{x} \rightarrow \alpha_{x}(x=0, y=0, z), \alpha_{y} \rightarrow \alpha_{y}(x=0, y=0, z)$, as $\varepsilon \rightarrow 0$ (and thus are independent of $\theta$ ), and since the integrals of the trigonometric functions of over the circle vanish. In addition,

$$
\lim _{\varepsilon \rightarrow 0} \int_{S_{\varepsilon}} \frac{b}{2 \pi} \alpha_{z} \mathrm{~d} \theta=b \alpha_{z}(0,0, z)
$$

and one concludes that

$$
\partial T_{\varphi}(\alpha)=b \int_{-\infty}^{\infty} \alpha_{z}(0,0, z) \mathrm{d} z
$$

If we assign the natural orientation to $L=\{(0,0)\} \times \mathbb{R} \subset \mathbb{R}^{3}$, we may use $T_{L}$ to denote the 1-current given by

$$
T_{L}(\alpha)=\int_{L} \iota_{L}^{*}(\alpha)
$$

Here $\iota_{L}^{*}: L \rightarrow \mathbb{R}^{3}$ is the natural inclusion so that for any 1 -form $\alpha=\alpha_{x} \mathrm{~d} x+$ $\alpha_{y} \mathrm{~d} y+\alpha_{z} \mathrm{~d} z, \iota_{L}^{*}(\alpha)=\alpha_{z} \mathrm{~d} z$. Thus, we may write the current as

$$
\partial T_{\varphi}=b T_{L}
$$


Remark 3. Using the same notation as above, consider the case where instead of $\varphi$ given in (7.2) one has the 1-form $\varphi^{\prime}$ given by

$$
\varphi^{\prime}=-\frac{b}{2 \pi} \mathrm{d} \theta
$$

Since $\varphi^{\prime}$ is annihilated by the vector space spanned by the base vectors $\partial / \partial r$ and $\partial / \partial z$, the layers induced by $\varphi^{\prime}$ look like the pages of a book spread evenly in all directions. If we follow the same steps as above we obtain

$$
\varphi^{\prime} \wedge \alpha=-\frac{b}{2 \pi}\left(\alpha_{r} \mathrm{~d} \theta \wedge \mathrm{d} r+\alpha_{z} \mathrm{~d} \theta \wedge \mathrm{d} z\right),
$$

so that

$$
\iota^{*}\left(\varphi^{\prime} \wedge \alpha\right)=-\frac{b}{2 \pi} \alpha_{z} \mathrm{~d} \theta \wedge \mathrm{d} z
$$

If follows that $\partial T_{\varphi^{\prime}}=\partial T_{\varphi}$. This observation may be viewed as follows. Let $T_{\mathrm{d} z}$ be the current induced by the form $\mathrm{d} z$. Then, since $\mathrm{d}^{2} z=0, \partial T_{\mathrm{d} z}=0$. Since $T_{\varphi}=T_{\varphi^{\prime}}-(b / 2 \pi) T_{\mathrm{d} z}$, it follows that $\partial T_{\varphi}=\partial T_{\varphi^{\prime}}$. Alternatively, one may envisage a smooth twist of $\mathbb{R}^{3}$ about the $z$-axis under which the book is deformed into the book. Since our objects are invariant under diffeomorphisms, both layering structures have the same dislocations. Thus for example, a similar observation will hold if the pages of the book are not plane but are bent perpendicularly to the $z$-axis forming the shape of a whirlpool.

7.4. The Frank Rules for Dislocations. If the 2-form $\psi$ describes the continuous distribution of dislocations, the Frank rules are induced by the compatibility condition $\mathrm{d} \psi=0$. For the singular case, if an $(n-2)$-current $R$ represents the geometry of the dislocations, the compatibility condition that induces Frank's rules is $\partial R=0$.

For example, let $\mathcal{S}$ be an $(n-1)$-dimensional submanifold with boundary of $\mathcal{M}$ and consider the the $(n-2)$-current $R$ so that

$$
R(\psi)=\int_{\partial \mathcal{S}} u \psi
$$

for some given differentiable function $u$ defined on $\partial \mathcal{S}$. then, the boundary $\partial R$ is given by

$$
\begin{aligned}
\partial R(\alpha) & =\int_{\partial \mathcal{S}} u \mathrm{~d} \alpha \\
& =\int_{\partial \mathcal{S}} \mathrm{d}(u \alpha)-\int_{\partial \mathcal{S}} \mathrm{d} u \wedge \alpha, \\
& =\int_{\partial^{2} \mathcal{S}} u \alpha-\int_{\partial \mathcal{S}} \mathrm{d} u \wedge \alpha, \\
& =-\int_{\partial \mathcal{S}} \mathrm{d} u \wedge \alpha
\end{aligned}
$$


Here, $\alpha$ is any $(n-3)$-form so for the three dimensional case $\alpha$ is any smooth function of compact support. Since compatibility imposes the condition $\partial R=0$, it follows that for $R$ to be a dislocation current, the function $u$ must be constant on $\partial S$ which is Frank's first rule.

\section{INCLINATIONS AND DISCLINATIONS}

Disclinations are viewed here as defects in the arrangements of 1-dimensional subspaces, or directors. As in [Fra58] and [Cha77], this field may indicate the inclinations of the optical axes of liquid crystals. The interpretation of disclinations as defects in the orientations of the Bravais planes (e.g., [KA75]) may be viewed in some cases as defects in the arrangements of the normal vectors to the respective Bravais planes. Such cases can be described using the framework outlined below.

Thus, disclinations are represented as boundaries of currents of order 1 . In the smooth case such a current is represented by an $(n-1)$-form $\varphi$, the inclination form, and the structure of the disclinations is given by the $n$ form $\mathrm{d} \varphi$. It is noted that any $(n-1)$-form is decomposable. (See [Ste83, Section 1.V], and [SR03] for a continuum mechanical application.) The induced distribution is necessarily involutive and the 1-dimensional integral submanifolds to which the directors are tangent may be easily constructed as follows.

At each point $x \in \mathcal{M}$ where $\varphi(x) \neq 0, \varphi(x)$ determines a unique 1dimensional subspace $\mathbf{W}_{x}$ of the tangent space $T_{x} \mathcal{M}$ by $\left.v\right\lrcorner \varphi(x)=0$ for each $v \in \mathbf{W}_{x}$. The collection of subspaces $\mathbf{W}_{x}$ forms a 1-dimensional distribution. The 1-dimensional subspace $\mathbf{W}_{x}$ may be determined as follows. Let $\theta$ be a volume element on $\mathcal{M}$. Locally, $\theta$ may be represented in the form

$$
\theta=\theta_{0} \mathrm{~d} x^{1} \wedge \cdots \wedge \mathrm{d} x^{n}
$$

for a positive real valued function $\theta_{0}$ and $\varphi$ may be represented locally in the form

$$
\varphi=\sum_{i=1}^{n} \varphi_{1 \ldots \hat{i} \ldots n} \mathrm{~d} x^{1} \wedge \cdots \wedge \widehat{\mathrm{d} x^{i}} \wedge \cdots \wedge \mathrm{d} x^{n}
$$

where a "hat" indicates the omission of an element. Then, there is a unique tangent vector $u$ such that $u\lrcorner \theta=\varphi$. If a vector $u$ is represented by $u=$ $\sum_{i} u^{i} \partial / \partial x^{i}$, then, $\left.u\right\lrcorner \theta$ is represented by

$$
u\lrcorner \theta=\sum_{i=1}^{n}(-1)^{i-1} \theta_{0} u^{i} \mathrm{~d} x^{1} \wedge \cdots \wedge \widehat{\mathrm{d} x^{i}} \wedge \cdots \wedge \mathrm{d} x^{n} .
$$

Thus, as $\theta_{0} \neq 0$, there is always a vector field $u$ satisfying $\left.u\right\lrcorner \theta=\varphi$ and its components are given locally by

$$
u^{i}=(-1)^{i-1} \frac{\varphi_{1 \ldots \hat{i} \ldots n}}{\theta_{0}} \text {. }
$$

If we select a different volume element, the only parameter that will change in the equation above will be the positive number $\theta_{0}$ and so the resulting 
vector will be in the same one dimensional subspace. Thus, the form $\varphi$ determines a unique oriented 1-dimensional subspace $\mathbf{U}_{x}$ at each $x$ such that $\varphi(x) \neq 0$. If no particular orientation is chosen on $\mathcal{M}$ no orientation will be induced on $\mathbf{U}_{x}$. The space $\mathbf{W}_{x}$ and $\mathbf{U}_{x}$ are isomorphic. Let $\theta$ be a volume elements and $u$ the vector such that $\varphi=u\lrcorner \theta$. Then, any nonzero $v \in \mathbf{U}_{x}$ is of the form $v=a u, a \neq 0$. Thus, $\left.\left.\left.\left.v\right\lrcorner(u\lrcorner \theta\right)=a u\right\lrcorner(u\lrcorner \theta\right)=0$, because $\theta\left(u, u, v_{3}, \ldots, v_{n}\right)=0$ for any collection of vectors $v_{3}, \ldots, v_{n}$.

For an $(n-1)$-form $\varphi$ we interpret the distribution $\mathbf{W}$ of 1-dimensional subspaces of the tangent space as indicating the inclinations of the directors in the body. Multiplying the form $\varphi$ by a positive number will affect the "density" of the directors.

Unlike the case of Bravais hyperplanes, inclination fields are always involutive, i.e., at each point $x \in \mathcal{M}$ there is a curve $c_{x}:(-\varepsilon, \varepsilon) \rightarrow \mathcal{M}, \varepsilon>0$, such that $c_{x}(0)=x$ and the tangent vector to the curve satisfies

$$
\left.\frac{\mathrm{d} c_{x}}{\mathrm{~d} t}\right|_{t=0} \in \mathbf{W}_{x} \text {. }
$$

Since we have assumed that the form $\varphi$ is differentiable, it follows that for a choice of a smooth volume element $\theta$, the representing vector field $u$ is differentiable. Hence, the theorems on the existence and uniqueness of the solutions of ordinary differential equations imply the existence of the integral lines to the vector field $u$,i.e., at each point $x \in \mathcal{M}$ there is a curve $c_{x}:(-\varepsilon, \varepsilon) \rightarrow \mathcal{M}, \varepsilon>0$, such that $c_{x}(0)=x$ and the tangent vector to the curve

$$
\left.\frac{\mathrm{d} c_{x}}{\mathrm{~d} t}\right|_{t=0}=u(x) .
$$

An inclination form may be integrated over $(n-1)$-dimensional submanifolds of $\mathcal{M}$. Let $\mathcal{S}$ be an oriented $(n-1)$-dimensional submanifold of $\mathcal{M}$. Then,

$$
\Phi_{\mathcal{S}}=\int_{\mathcal{S}} \varphi
$$

is interpreted as the total amount of directors penetrating the surface $\mathcal{S}$. It should be noted that $\Phi_{\mathcal{S}}$ depends on the orientation of $\mathcal{S}$ and that the restriction of $\varphi$ to a point in $\mathcal{S}$ may be of the same orientation as $\mathcal{S}$ or the inverse orientation. Thus, for a nonvanishing inclination form, the total $\Phi_{\mathcal{S}}$ may vanish which implies that each of the integral lines penetrates $\mathcal{S}$ in one orientation the same number of times that it penetrates $\mathcal{S}$ in the opposite orientation.

For the inclination $(n-1)$-form $\varphi$, the distribution of smooth disclinations induced is the exterior derivative, the $n$-form $\mathrm{d} \varphi$. Thus, for a $n$ dimensional submanifold with boundary $\mathcal{B} \subset \mathcal{M}$, letting $\mathcal{S}=\mathcal{B}$ in (8.6), $\Phi_{\partial \mathcal{B}}$ is interpreted as the total amount of directors that penetrate $\partial \mathcal{B}$.

Stokes's theorem implies immediately that

$$
\Phi_{\partial \mathcal{B}}=\int_{\mathcal{B}} \mathrm{d} \varphi,
$$


so that $\Phi_{\partial \mathcal{B}}$ is the integral of the disclination field over $\mathcal{B}$. Figuratively speaking, the disclination field represents the source term for the directors.

It is observed that for any given vector field one can label the integral lines by a submanifold of dimension $n-1$ of initial conditions (see AMR88, pp. 246-247]). However, the vector fields induced by $\varphi$ depend on the choice of volume element $\theta$. Thus, such labeling is not unique and the presence of disclinations will reflected by $\mathrm{d} \varphi$.

An inclination $(n-1)$-form $\varphi$ induces a de Rham 1-current $T_{\varphi}$ as in (4.4). In the non-smooth case, we replace the inclination 1-form $\varphi$ and the current it induces by a general inclination 1 -current $T$. Inclination currents that are not given in terms smooth $(n-1)$-forms represent singular, or concentrated, director fields as the examples below illustrate.

Example 4. A non-coherent interface 1. Consider the locally integrable $(n-1)$-form $\varphi$ in $\mathbb{R}^{n}$ given by

$$
\varphi(x)= \begin{cases}\mathrm{d} x^{1} \wedge \cdots \wedge \mathrm{d} x^{n-1}, & \text { for } x \in \overline{\mathbb{R}}^{n+}, \\ a \mathrm{~d} x^{1} \wedge \cdots \wedge \mathrm{d} x^{n-1}, & \text { for } x \in \mathbb{R}^{n-},\end{cases}
$$

where $a \in \mathbb{R}$ is a constant, $\mathbb{R}^{n-}=\left\{x \in \mathbb{R}^{n} \mid x^{n}<0\right\}$, and $\overline{\mathbb{R}}^{n+}=\left\{x \in \mathbb{R}^{n} \mid\right.$ $\left.x^{n} \geqslant 0\right\}$. The inclination form $\varphi$ induces a 1 -current $T_{\varphi}$ by

$$
T_{\varphi}(\omega)=\int_{\mathbb{R}^{n}} \varphi \wedge \omega .
$$

Clearly, the 1-dimensional subspace spanned by $\partial / \partial x^{n}$ annihilates $\varphi(x)$ for all $x$ for which $x^{n} \neq 0$. Thus, the directors are aligned in the $x^{n}$ direction.

For any smooth compactly supported 0 -form $\alpha$ in $\mathbb{R}^{n}$,

$$
\begin{aligned}
\partial T_{\varphi}(\alpha) & =\int_{\mathbb{R}^{n}} \varphi \wedge \mathrm{d} \alpha, \\
& =\int_{\mathbb{R}^{n-}} \varphi \wedge \mathrm{d} \alpha+\int_{\overline{\mathbb{R}}^{n+}} \varphi \wedge \mathrm{d} \alpha, \\
& =(-1)^{n-1}\left[\int_{\mathbb{R}^{n-}} \mathrm{d}(\alpha \varphi)-\int_{\mathbb{R}^{n-}} \alpha \mathrm{d} \varphi+\int_{\mathbb{R}^{n+}} \mathrm{d}(\alpha \varphi)-\int_{\mathbb{R}^{n+}} \alpha \mathrm{d} \varphi\right], \\
& =(-1)^{n-1}\left[\int_{\partial \mathbb{R}^{n-}} \alpha \varphi+\int_{\partial \mathbb{R}^{n+}} \alpha \varphi\right]
\end{aligned}
$$

where in the third line we used (2.11). Let $P$ be the hyperplane in $\mathbb{R}^{n}$ defined by $x^{n}=0$ oriented such that $P=\partial \mathbb{R}^{n-}=-\partial \mathbb{R}^{n+}$ so that $\theta_{P}=$ $\mathrm{d} x^{1} \wedge \cdots \wedge \mathrm{d} x^{n-1}$ is the natural volume element on $P$. Let $T_{P}$ be the $0-$ current given by

$$
T_{P}(\alpha)=\int_{P} \alpha \theta_{P}
$$

We conclude that

$$
\partial T_{\varphi}=(-1)^{n-1}(a-1) T_{P}
$$


which is interpreted as a concentrated source of directors of magnitude $a-1$ which is distributed over the $x^{1}, \ldots, x^{n-1}$ hyperplane.

Example 5. A non-coherent interface 2. Consider the locally integrable 1 -form $\varphi$ in $\mathbb{R}^{n}$ given by

$$
\varphi(x)= \begin{cases}\varphi_{2}, & \text { for } x \in \overline{\mathbb{R}}^{n+}, \\ \varphi_{1}, & \text { for } x \in \mathbb{R}^{n-},\end{cases}
$$

where $\varphi_{1}$ and $\varphi_{2}$ are uniform $(n-1)$-forms in $\mathbb{R}^{n-}$ and $\overline{\mathbb{R}}^{n+}$, respectively. Letting $T_{\varphi}$ be the 1-current defined by

$$
T_{\varphi}(\omega)=\int_{\mathbb{R}^{n}} \varphi \wedge \omega,
$$

it follows from (8.10) that

$$
\partial T_{\varphi}(\alpha)=\int_{P} \alpha\left(\varphi_{1}-\varphi_{2}\right)
$$

We conclude that the disclination current vanishes if $\varphi_{1}$ and $\varphi_{2}$ have the same restriction to $P$, i.e., both forms have the same component relative to $\mathrm{d} x^{1} \wedge \cdots \wedge \mathrm{d} x^{n-1}$. In particular, let $v$ be a vector parallel to the $x^{1}, \ldots, x^{n-1}$ plane. Then, $\left.\varphi_{2}=v\right\lrcorner \mathrm{d} x^{1} \wedge \cdots \wedge \mathrm{d} x^{n}$ is annihilated by the 1-dimensional space spanned by $v$ and all the components of $\varphi_{2}$ that do not vanish correspond to basis elements of the form

$$
\mathrm{d} x^{1} \wedge \cdots \wedge \widehat{\mathrm{d} x^{k}} \wedge \cdots \wedge \mathrm{d} x^{n}, \quad k=1, \ldots, n-1 .
$$

In this case, the directors corresponding the $\varphi_{2}$ do not intersect the $x^{1}, \ldots, x^{n-1}$ plane, the component of $\varphi_{2}$ relative to $\mathrm{d} x^{1} \wedge \cdots \wedge \mathrm{d} x^{n-1}$ vanishes, and

$$
\partial T_{\varphi}(\alpha)=\int_{P} \alpha \varphi_{1}
$$

Example 6. An edge disclination. Let $L$ be a connected and oriented 1dimensional submanifold with a boundary of $\mathcal{M}$. Then, $L$ induces a 1current $T_{L}$ by

$$
T_{L}(\omega)=\int_{L} \omega,
$$

for all compactly supported smooth 1-forms $\omega$ in $\mathcal{M}$. Using Stokes's theorem, one has

$$
\partial \mathrm{T}_{L}(\alpha)=\int_{L} \mathrm{~d} \alpha=\int_{\partial L} \alpha
$$

Evidently, as $\partial L$ is a 0 -dimensional submanifold, and assuming it is not empty, it may contain one or two points, each having either a positive or a negative orientation while the other point, if exists, has the opposite orientation.

In the case where $\partial L$ contains one point $x_{1}$ and assuming its orientation is positive, one has $\partial L(\alpha)=\alpha\left(x_{0}\right)$, representing an edge disclination originating at $x_{0}$. This will be the situation if $\mathcal{M}=(-1,1)^{3} \subset \mathbb{R}^{3}$ and $L=$ 
$\{(0,0, z) \mid-1<z \leqslant 0\}$ so that $x_{1}=(0,0,0)$. In this case the disclination does not terminate inside the body. In the case where $\partial L$ contains also the additional point $x_{2}$ having a negative orientation, $\partial L(\alpha)=\alpha\left(x_{1}\right)-\alpha\left(x_{2}\right)$ and the disclination terminates at $x_{2}$.

Example 7. Directors emanating from a singular line. Using the notation introduced in Section 7.3 on screw dislocations, consider the inclination $n-1=2$-form $\varphi$ defined on $D \subset \mathbb{R}^{3}$ by

$$
\varphi=\mathrm{d} \theta \wedge \mathrm{d} z .
$$

The inclination form induces an inclination 1-current $T$ on $\mathbb{R}^{3}$ by the right had side of (7.6). It is noted that in its domain of definition, $\mathrm{d} \varphi=0$.

To compute the disclination 0 -current $\partial T$, one observes that for any smooth function $\alpha$, compactly supported in $\mathbb{R}^{3},(2.11)$ implies that

$$
\begin{aligned}
\partial T(\alpha) & =\int_{D} \mathrm{~d}(\alpha \varphi)-\int_{D} \alpha \mathrm{d} \varphi, \\
& =\lim _{\varepsilon \rightarrow 0} \int_{D_{\varepsilon}} \mathrm{d}(\alpha \varphi), \\
& =\lim _{\varepsilon \rightarrow 0} \int_{\partial D_{\varepsilon}} \iota^{*}(\alpha \varphi), \\
& =\lim _{\varepsilon \rightarrow 0} \int_{\partial D_{\varepsilon}} \alpha \mathrm{d} \theta \wedge \mathrm{d} z .
\end{aligned}
$$

In analogy with the computations of Section 7.3 , one obtains

$$
\partial T(\alpha)=2 \pi \int_{z=-\infty}^{\infty} \alpha(0,0, z) \mathrm{d} z
$$

which we may write as

$$
\partial T=2 \pi T_{L}\llcorner\mathrm{~d} z .
$$

Thus, we have a uniform distribution of directors' source along the $z$-axis.

\section{Kinematics of Defect Distributions}

In this section we consider the kinematics of the material structure and the distribution of defects. Noting that material structure and the associated defects are viewed here as intrinsic to a body and unrelated to the kinematics of the body in space, in the following two subsections we consider the motion of material structure and defects resulting from a family of diffeomorphisms of the body. (See [FS13] for another application of the same mathematical notions.) In other words, the material structure, as represented by a smooth form and its exterior derivative or a de Rham current and its boundary, are carried with material diffeomorphisms. In contrast, the last subsection proposes an example for an evolution of a continuously distributed material structure to a singular one using a process which is the opposite of smoothing. 
9.1. Smooth Evolutions of Structure Forms and Continuously Distributed Defects. In order to study the deformation of structure forms and currents, we consider the following setting. It is assumed that we are given a time dependent flow, or a smooth evolution operator, $\Phi: \mathcal{I}^{2} \times \mathcal{M} \rightarrow \mathcal{M}$ in the interval $\mathcal{I}=[a, b] \subset \mathbb{R}$. That is, for each time instances $t$ and $\tau$, with $t \leqslant \tau$, $t, \tau \in \mathcal{I}, \Phi_{\tau, t}: \mathcal{M} \rightarrow \mathcal{M}$ is a diffeomorphism and $\Phi_{\tau, t} \circ \Phi_{t, s}=\Phi_{\tau, s}$. For $t \geqslant$ $\tau, \Phi_{\tau, t}=\Phi_{t, \tau}^{-1}$, which implies that $\Phi_{t, t}=I_{\mathcal{M}}$, the identity diffeomorphism. Evidently, the flow induces a smooth homotopy $h:[a, b] \times \mathcal{M} \rightarrow \mathcal{M}$ by $h(t, x)=h_{t}(x)=\Phi_{t, a}(x)$ so that $\Phi_{\tau, t}=h_{\tau} \circ h_{t}^{-1}$. The time dependent flow induces a time dependent vector field $w: \mathcal{I} \times \mathcal{M} \rightarrow T \mathcal{M}$ by setting

$$
w(t, x)=\left.\frac{\partial}{\partial \tau}\right|_{\tau=t} \Phi_{\tau, t}(x)=\left.\frac{\partial}{\partial \tau}\right|_{\tau=t} h_{\tau}\left(h_{t}^{-1}(x)\right),
$$

that is, $w(t, x)$ is the tangent at the time $\tau=t$ to the curve

$$
c_{t, x}(\tau)=\Phi_{\tau, t}(x)=h_{\tau}\left(h_{t}^{-1}(x)\right),
$$

starting at $x$ at time $t$ (see for example [AMR88, p. 283]). Conversely, the flow is the solution of the differential equation

$$
w\left(s, \Phi_{s, t}(x)\right)=\left.\frac{\partial}{\partial \tau}\right|_{\tau=s} c_{t, x}(\tau)=\left.\frac{\partial}{\partial \tau}\right|_{\tau=s} \Phi_{\tau, t}(x)=\left.\frac{\partial}{\partial \tau}\right|_{\tau=s} h_{\tau}\left(h_{t}^{-1}(x)\right) .
$$

Alternatively, setting $t=a$, the differential equation may be expressed as

$$
w\left(s, h_{s}(x)\right)=\left.\frac{\partial}{\partial \tau}\right|_{\tau=s} h_{\tau}(x) .
$$

Each diffeomorphism $\Phi_{\tau, t}$ induces the pullback of forms $\Phi_{\tau, t}^{*}$ from Image $h_{\tau}$ onto Image $h_{t}$. In particular, if $\omega$ is an $r$-form with compact support in $\mathcal{M}$, the same holds for its pullback $\Phi_{\tau, t}^{*} \omega$.

Let $\varphi$ be a (time independent) differentiable, material structure $(n-r)$ form. Then, for each $t \in \mathcal{I}$, the flow induces a time dependent $(n-r)$-form $\Phi_{\tau, t}^{*} \varphi$ and in particular the form $h_{t}^{*} \varphi$. It is also recalled that for any $(n-r)-$ dimensional submanifold $\mathcal{S} \subset \mathcal{M}$, and a form $\varphi$ having a compact support, one has [AMR88, p. 466]

$$
\int_{\mathcal{S}} h_{t}^{*} \varphi=\int_{h_{t}\{\mathcal{S}\}} \varphi
$$

This will hold in the particular case where the submanifold $\mathcal{S}$ is compact so that the restriction of $h_{t}^{*} \varphi$ to $\mathcal{S}$ has a compact support. It is evident from the above observations that rather than $h_{t}^{*} \varphi$, it is the pushforward $h_{t}^{-*} \varphi:=h_{t}^{*-1} \varphi=h_{t}^{-1 *} \varphi$ that represents the evolution of the structure form. Specifically, replacing $h_{t}$ by $h_{t}^{-1}$ and $\mathcal{S}$ by $h_{t}\{\mathcal{S}\}$ above, one has

$$
\int_{h_{t}\{\mathcal{S}\}} h_{t}^{-*} \varphi=\int_{\mathcal{S}} \varphi
$$


which indicates how the evolution of the structure form follows the evolution $h_{t}\{\mathcal{S}\}$ of the submanifold. It is emmphasized that we regard the flow $\Phi$ to be associated with the structure of matter only and has nothing to do with the motion in space of the material points belonging to the body.

It is recalled that for each differentiable mapping $f: \mathcal{M} \rightarrow \mathcal{N}$ between a manifold $\mathcal{M}$ and a manifold $\mathcal{N}$, and a differentiable form $\varphi$, one has $f^{*}(\mathrm{~d} \varphi)=\mathrm{d}\left(f^{*} \varphi\right)$. Thus, in our setting, $h_{t}^{-*}(\mathrm{~d} \varphi)=\mathrm{d}\left(h_{t}^{-*} \varphi\right)$, that is, the smooth field of defects induced by $h_{t}^{-*} \varphi$ is obtained by the pushforward of the field of defects induced by $\varphi$.

We also note that as $f^{*}(\alpha \wedge \beta)=f^{*} \alpha \wedge f^{*} \beta$ [AMR88, p. 420]. Thus, if $T_{\varphi}$ is the $r$-current on $\mathcal{M}$ induced by $\varphi$, then for any $r$-form $\omega$ having a compact support on $\mathcal{M}$,

$$
\begin{aligned}
T_{\varphi}(\omega) & =\int_{\mathcal{M}} \varphi \wedge \omega, \\
& =\int_{\mathcal{M}} h_{t}^{-*}(\varphi \wedge \omega), \\
& =\int_{\mathcal{M}} h_{t}^{-*} \varphi \wedge h_{t}^{-*} \omega .
\end{aligned}
$$

It is concluded therefore that

$$
T_{\varphi}\left(h_{t}^{*} \omega\right)=T_{h_{t}^{-*} \varphi}(\omega) .
$$

Next, we would like to compute the rate at which the structure form evolves under the flow. In general, using the Lie derivative $\mathcal{L}_{w_{\tau}} \omega$ of a form $\omega$ relative to the vector field $w_{\tau}$ associated with the flow $\Phi_{\tau, t}$, one has [AMR88, p. 372]

$$
\left.\frac{\partial}{\partial \tau}\right|_{\tau=s} \Phi_{\tau, t}^{*} \omega=\Phi_{s, t}^{*}\left(\mathcal{L}_{w_{s}} \omega\right)
$$

In particular, for $s=t$ and for $t=a$, the relation above specializes to

$$
\left.\frac{\partial}{\partial \tau}\right|_{\tau=t} \Phi_{\tau, t}^{*} \omega=\mathcal{L}_{w_{t}} \omega,\left.\quad \frac{\partial}{\partial \tau}\right|_{\tau=s} h_{\tau}^{*} \omega=h_{s}^{*}\left(\mathcal{L}_{w_{s}} \omega\right) .
$$

It is noted that the last two equations hold pointwise. It follows that for each $x \in \mathcal{M}$,

$$
\Phi_{\tau_{2}, t}^{*} \omega(x)-\Phi_{\tau_{1}, t}^{*} \omega(x)=\int_{\tau_{1}}^{\tau_{2}} \Phi_{\tau, t}^{*}\left(\mathcal{L}_{w_{\tau}} \omega\right)(x) \mathrm{d} \tau .
$$

For the rate of change of the pushforward of the structure form, one has to use in the equations above the time dependent vector field $w^{-1}$ associated with the flow $\Phi_{\tau, t}^{-1}$. According to (9.3), it is given by

$$
w^{-1}\left(s, \Phi_{s, t}^{-1}(x)\right)=\left.\frac{\partial}{\partial \tau}\right|_{\tau=s} \Phi_{\tau, t}^{-1}(x)=\left.\frac{\partial}{\partial \tau}\right|_{\tau=s} h_{t}\left(h_{\tau}^{-1}(x)\right) .
$$


Since $\Phi_{\tau, t}^{-1} \circ \Phi_{\tau, t}$ is the identity on $\mathcal{M}$,

$$
\begin{aligned}
0 & =\left.\frac{\partial}{\partial \tau}\right|_{\tau=s} \Phi_{\tau, t}^{-1}\left(\Phi_{\tau, t}(x)\right) \\
& =\left.\frac{\partial}{\partial \tau}\right|_{\tau=s} \Phi_{\tau, t}^{-1}\left(\Phi_{s, t}(x)\right)+T \Phi_{s, t}^{-1}\left(\left.\frac{\partial}{\partial \tau}\right|_{\tau=s} \Phi_{\tau, t}(x)\right) \\
& =w^{-1}\left(s, \Phi_{s, t}^{-1}\left(\Phi_{s, t}(x)\right)\right)+T \Phi_{s, t}^{-1}\left(w\left(s, \Phi_{s, t}(x)\right)\right) .
\end{aligned}
$$

It is implied that

$$
w^{-1}(s, x)=-T \Phi_{s, t}^{-1}\left(w\left(s, \Phi_{s, t}(x)\right)\right)
$$

or,

$$
w_{s}^{-1}=-T \Phi_{s, t}^{-1} \circ\left(w_{s} \circ \Phi_{s, t}\right)
$$

and in particular,

$$
w^{-1}(t, x)=-w(t, x) .
$$

The rate of in which the structure form $\varphi$ evolves is therefore

$$
\begin{aligned}
\left.\frac{\partial}{\partial \tau}\right|_{\tau=s} \Phi_{\tau, t}^{-*}(\varphi) & =\Phi_{s, t}^{-*}\left(\mathcal{L}_{w_{s}^{-1}} \varphi\right), \\
& =-\Phi_{s, t}^{-*}\left(\mathcal{L}_{T \Phi_{s, t}^{-1} \circ\left(w_{s} \circ \Phi_{s, t}\right.} \varphi\right) .
\end{aligned}
$$

We also recall [AMR88, p. 361] that in general, for a diffeomorphism $f$ : $\mathcal{M} \rightarrow \mathcal{N}$, a vector field $w$ and a form $\omega$,

$$
f^{*}\left(\mathcal{L}_{T f(w)} \omega\right)=\mathcal{L}_{w} f^{*} \omega, .
$$

Substituting $\Phi_{\tau, t}^{-1}$ for $f$, one has

$$
\left.\frac{\partial}{\partial \tau}\right|_{\tau=s} \Phi_{\tau, t}^{-*}(\varphi)=-\mathcal{L}_{w_{s}}\left(\Phi_{s, t}^{-*} \varphi\right)
$$

In particular,

$$
\left.\frac{\partial}{\partial \tau}\right|_{\tau=t} \Phi_{\tau, t}^{-*}(\varphi)=-\mathcal{L}_{w_{t}} \varphi
$$

is the rate in which the structure form evolves.

Since the Lie derivative commutes with exterior differentiation [AMR88, p. 428], the rate of change of the distribution of defects is the exterior derivative of the rate of change of the structure form, i.e.,

$$
\left.\frac{\partial}{\partial \tau}\right|_{\tau=t} \Phi_{\tau, t}^{-*}(\mathrm{~d} \varphi)=-\mathcal{L}_{w_{t}} \mathrm{~d} \varphi=-\mathrm{d} \mathcal{L}_{w_{t}} \varphi=\mathrm{d}\left(\left.\frac{\partial}{\partial \tau}\right|_{\tau=t} \Phi_{\tau, t}^{-*}(\varphi)\right) .
$$


9.2. Evolutions of General Structure Currents and Defects. We wish to extend the kinematic analysis for smooth deformations of structure forms and continuously distributed defects to general, possibly singular currents. The way this is done is suggested by (9.8) where we observed that for a current $T_{\varphi}$ induced by a smooth form $\varphi$, the current induced by the evolving form $h_{t}^{-*} \varphi$ satisfies $T_{h_{t}^{-*} \varphi}(\omega)=T_{\varphi}\left(h_{t}^{*} \omega\right)$.

Since for each time $t, h_{t}$ is a diffeomorphism of $\mathcal{M}$, given any smooth form $\omega$ having a compact support in $\mathcal{M}$, the pullback $h_{t}^{*} \omega$ has a compact support in $\mathcal{M}$, also. In fact, $h_{t}^{*}$ is a continuous, linear operator on the space of smooth forms with compact supports in $\mathcal{M}$. Thus, for a diffeomorphism $f$, the dual operator, the pushforward of currents (or images of currents [dR84, p. 47]), $f_{*}$, is defined by

$$
\left(f_{*} T\right)(\omega)=T\left(f^{*} \omega\right) .
$$

Thus, the evolution of a structure current $T$ under the flow is described by the evolution $h_{t *} T$ for which the analysis above is a special case.

It is observed that

$$
\begin{aligned}
\partial\left(h_{t *} T\right)(\psi) & =h_{t *} T(\mathrm{~d} \psi), \\
& =T\left(h_{t}^{*}(\mathrm{~d} \psi)\right), \\
& =T\left(\mathrm{~d}\left(h_{t}^{*} \psi\right)\right), \\
& =\partial T\left(h_{t}^{*} \psi\right), \\
& =\left(h_{t *}(\partial T)\right)(\psi),
\end{aligned}
$$

and so,

$$
\partial\left(h_{t *} T\right)=h_{t *}(\partial T)
$$

We conclude that the evolution of the defects follows the evolution of structure current, consistently.

To present a typical example for the evolution of a current which is not induced by a smooth structure form, consider the $r$-current $T_{\mathcal{S}}$ induced by an $r$-dimensional submanifold with boundary $\mathcal{S}$ of $\mathcal{M}$ as in 4.5). Then,

$$
\begin{aligned}
h_{t *} T_{\mathcal{S}}(\omega) & =T_{\mathcal{S}}\left(h_{t}^{*} \omega\right), \\
& =\int_{\mathcal{S}} h_{t}^{*} \omega, \\
& =\int_{h_{t}\{\mathcal{S}\}} \omega,
\end{aligned}
$$

and we conclude that

$$
h_{t *} T_{\mathcal{S}}=T_{h_{t}\{\mathcal{S}\}},
$$

i.e., the image of the structure current induced by $\mathcal{S}$ is the structure current induced by $h_{t}\{\mathcal{S}\}$. As expected, the defect current satisfies

$$
\partial\left(h_{t *} T_{\mathcal{S}}\right)=h_{t *}\left(\partial T_{\mathcal{S}}\right)=h_{t *}\left(T_{\partial \mathcal{S}}\right) .
$$


Next, we consider the rate of change of the pushforward of the structure current and the associated defect current. One has,

$$
\begin{aligned}
\left.\frac{\partial}{\partial \tau}\right|_{\tau=s}\left(\Phi_{\tau, t *} T(\omega)\right) & =\left.\frac{\partial}{\partial \tau}\right|_{\tau=s}\left(T\left(\Phi_{\tau, t}^{*} \omega\right)\right), \\
& =\lim _{\Delta \tau \rightarrow 0} \frac{T\left(\Phi_{\tau+\Delta \tau, t}^{*} \omega\right)-T\left(\Phi_{\tau, t}^{*} \omega\right)}{\Delta \tau}, \\
& =\lim _{\Delta \tau \rightarrow 0} T\left(\frac{\Phi_{\tau+\Delta \tau, t}^{*} \omega-\Phi_{\tau, t}^{*} \omega}{\Delta \tau}\right) .
\end{aligned}
$$

If

$$
\lim _{\Delta \tau \rightarrow 0} \frac{\Phi_{\tau+\Delta \tau, t}^{*} \omega-\Phi_{\tau, t}^{*} \omega}{\Delta \tau}
$$

exists in the sense of test forms (not merely pointwise), then, one may switch the order of the limit and the action of $T$ in the last line of (9.28) above. This is indeed the case (see [dR84, pp. 57-61], and also [GMS98, pp. 132-135], [Fed69, p. 363]). Hence, using (9.94 [9.10),

$$
\begin{aligned}
\left.\frac{\partial}{\partial \tau}\right|_{\tau=s}\left(\Phi_{\tau, t *} T(\omega)\right) & =T\left(\left.\frac{\partial}{\partial \tau}\right|_{\tau=s}\left(\Phi_{\tau, t}^{*} \omega\right)\right), \\
& =T\left(\Phi_{s, t}^{*}\left(\mathcal{L}_{w_{s}} \omega\right)\right), \\
& =\left(\Phi_{s, t *} T\right)\left(\mathcal{L}_{w_{s}} \omega\right) .
\end{aligned}
$$

It is noted that the Lie derivative operator on smooth forms with compact supports is linear and continuous, so that one may define its dual transformation $\mathcal{L}_{w}^{*}$ on the space of currents as

$$
\left(\mathcal{L}_{w}^{*} T\right)(\omega)=T\left(\mathcal{L}_{w} \omega\right)
$$

It is also recalled that Cartan's magic formula for the Lie derivative asserts that (e.g., [AMR88, p. 429])

$$
\left.\left.\mathcal{L}_{w} \omega=\mathrm{d}(w\lrcorner \omega\right)+w\right\lrcorner \mathrm{d} \omega,
$$

so that

$$
\begin{aligned}
\left(\Phi_{s, t *} T\right)\left(\mathcal{L}_{w_{s}} \omega\right) & \left.\left.=\left(\Phi_{s, t *} T\right)\left(\mathrm{d}\left(w_{s}\right\lrcorner \omega\right)\right)+\left(\Phi_{s, t *} T\right)\left(w_{s}\right\lrcorner \mathrm{d} \omega\right), \\
& \left.=\left(\partial \Phi_{s, t *} T\right)\left(w_{s}\right\lrcorner \omega\right)+\left(w_{s} \wedge \Phi_{s, t *} T\right)(\mathrm{d} \omega), \\
& =\left(w_{s} \wedge\left(\partial \Phi_{s, t *} T\right)+\partial\left(w_{s} \wedge \Phi_{s, t *} T\right)\right)(\omega) .
\end{aligned}
$$

Thus, the rate of change of the structure current may be expressed as

$$
\left.\frac{\partial}{\partial \tau}\right|_{\tau=s} \Phi_{\tau, t *} T=\mathcal{L}_{w_{s}}^{*} \circ \Phi_{s, t *}(T)=w_{s} \wedge\left(\partial \Phi_{s, t *} T\right)+\partial\left(w_{s} \wedge \Phi_{s, t *} T\right) .
$$

In particular,

$$
\left.\frac{\partial}{\partial \tau}\right|_{\tau=s} h_{\tau *} T=\mathcal{L}_{w_{s}}^{*} \circ h_{s *}(T)=w_{s} \wedge\left(\partial h_{s *} T\right)+\partial\left(w_{s} \wedge h_{s *} T\right),
$$


and

$$
\left.\frac{\partial}{\partial \tau}\right|_{\tau=t} \Phi_{\tau, t *} T=\mathcal{L}_{w_{t}}^{*}(T)=w_{t} \wedge \partial T+\partial\left(w_{s} \wedge T\right)
$$

9.3. Evolution of Smooth Distributions of Defects to Singular Ones. The theory of currents provides a mathematical construction that may be used to model the process at which a smooth distribution of defects evolves and they coalesce into a "macroscopic" singular defect.

Similarly to Schwartz distributions, the action of general currents can be approximated using currents induced by smooth forms through the process of regularization or smoothing (see [dR84, pp. 61-70], [Fed69, pp. 346348], [GMS98, pp. 505-511]). Specifically, given an $r$-current $T$, one can construct a family of smooth $(n-r)$-forms $\varphi_{\varepsilon}, \varepsilon \in(0,1]$ and corresponding $T_{\varepsilon}$ defined by

$$
T_{\mathcal{\varepsilon}}(\omega)=\int_{\mathcal{M}} \varphi_{\varepsilon} \wedge \omega
$$

so that

$$
\lim _{\varepsilon \rightarrow 0} T_{\varepsilon}(\omega)=T(\omega)
$$

In other words, $T_{\varepsilon}$ converge to $T$ weakly. Furthermore, recalling that $\partial T_{\varepsilon}$ is the current induced by $\mathrm{d} \varphi_{\varepsilon}$, i.e.,

$$
\partial T_{\mathcal{E}}(\psi)=(-1)^{n-r+1} T_{\mathrm{d} \varphi}(\psi)=(-1)^{n-r+1} \int_{\mathcal{M}} \mathrm{d} \varphi_{\mathcal{\varepsilon}} \wedge \psi,
$$

for each $(r-1)$-form $\psi$, the regularization process commutes with the boundary operator so that

$$
\lim _{\varepsilon \rightarrow 0} \partial T_{\varepsilon}(\psi)=(-1)^{n-r+1} \lim _{\varepsilon \rightarrow 0} \int_{\mathcal{M}} \mathrm{d} \varphi_{\varepsilon} \wedge \psi=\partial T(\psi) .
$$

Thus, setting $T_{0}=T$, and $t=1-\varepsilon$, rather than a formal mathematical approximation process, one could view the family $T_{t}, t \in[0,1]$, as an evolution process of structure currents in the time interval $[0,1]$ in which the smooth structure forms evolve into a discrete structure current. Finally, the fact that smoothing commutes with the boundary operator, implies that the smooth defect forms evolve into the defect current.

Acknowledgments. This work was partially supported by Perlstone Center for Aeronautical Engineering Studies at Ben-Gurion University.

\section{REFERENCES}

[AMR88] R. Abraham, J.E. Marsden, and T. Ratiu. Manifolds, Tensor Analysis. and Applications. Springer, 1988.

[Cer99] P. Cermelli. Material symmetry and singularities in solids. Proceedings of the Royal Society of London, A 455:299-322, 1999.

[Cha77] S. Chandrasekhar. Liquid Crystals. Cambridge University Press, 1977.

[dR84] G. de Rham. Differentiable Manifolds. Springer, 1984. 
[EC70] A.C. Eringen and W.D. Claus. A micromorphic approach to dislocation theory and its relation to several existing theories. In J.A. Simmons, R. de Wit, and R. Bullough, editors, Fundamental Aspects of Dislocation Theory, pages 1023-1040. U.S. National Bureau of Standards, 1970.

[EE07] M. Elzanowski and M. Epstein. Material Inhomogeneities and their Evolution. Springer, 2007.

[ES12] M. Epstein and R. Segev. Geometric aspects of singular dislocations. Mathematics and Mechanics of Solids, 2012.

[Fed69] H. Federer. Geometric Measure Theory. Springer, 1969.

[Fra58] F.C. Frank. 1. Liquid crystals. On the theory of liquid crystals. Discussions of the Faraday Society, 25:19-28, 1958.

[FS13] L. Falach and R. Segev. Reynolds transport theorem for smooth deformations of currents on manifolds. 2013. arXiv: 1312.7671[math-ph].

[GMS98] M. Giaquinta, G. Modica, and J. Soucek. Cartesian Currents in the Calculus of Variation I. Springer, 1998.

[KA75] E. Kroner and K.H. Anthony. Dislocations and disclinations in material structures: The basic topological concepts. Annual Review of Material Science, 5:43-72, 1975.

[Kon55] K. Kondo. Geometry of Elastic Deformation and incompatibility. Tokyo Gakujutsu Benken Fukyu-Kai, IC, 1955.

[LK06] S.A. Lurie and A.L. Kalamkarov. General theory of defects in continous media. International Journal of Solids and Structures, 43:91-111, 2006.

[Nol67] W. Noll. Materially uniform bodies with inhomogeneities. Archive for Rational mechanics and Analysis, 27:1-32, 1967.

[Sah84] D. Sahoo. Elastic continuum theories of lattice defects: a review. Bulletin of Materials Science, 6:775-798, 1984.

[SR03] R. Segev and G. Rodnay. Worldlines and body points associated with an extensive property. International Journal of Non-Linear Mechanics, 38:1-9, 2003.

[Ste83] S. Sternberg. Lectures on Differential Geometry. AMS Chelsea, 1983.

[Tou68] R.A. Toupin. Dislocated and oriented media. In Continuum Theory of Inhomogeneities in Simple Bodies, pages 9-24. Springer, 1968.

[Wan67] C.-C. Wang. On the geometric structure of simple bodies, a mathematical foundation for the theory of continuous distributions of dislocations. Archive for Rational Mechanics and Analysis, 27:33-94, 1967.

[Whi57] H. Whitney. Geometric Integration Theory. Princeton University Press, 1957.

University of CAlgary, CANADA; Ben-Gurion UniVersity of the NegeV, ISRAEL

E-mail address: mepstein@ucalgary.ca; rsegev@bgu.ac.il 